\title{
Costs of General Purpose Learning
}

\author{
John Case ${ }^{\text {a }}$ Keh-Jiann Chen ${ }^{\mathrm{b}}$ Sanjay Jain ${ }^{\mathrm{c}}$

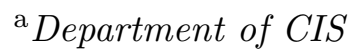 \\ University of Delaware \\ Newark, DE 19716 \\ USA \\ Email: case@cis.udel.edu \\ b Institute for Information Sciences \\ Academica Sinica \\ Taipei, 15, Taiwan \\ Republic of China \\ c School of Computing \\ National University of Singapore \\ Singapore 119260 \\ Email:sanjay@comp.nus.edu.sg
}

\begin{abstract}
Leo Harrington surprisingly constructed a machine which can learn any computable function $f$ according to the following criterion (called $\mathbf{B} \mathbf{c}^{*}$-identification). His machine, on the successive graph points of $f$, outputs a corresponding infinite sequence of programs $p_{0}, p_{1}, p_{2}, \ldots$, and, for some $i$, the programs $p_{i}, p_{i+1}, p_{i+2}, \ldots$ each compute a variant of $f$ which differs from $f$ at only finitely many argument places. A machine with this property is called general purpose. The sequence $p_{i}, p_{i+1}, p_{i+2}, \ldots$ is called a final sequence.
\end{abstract}

For Harrington's general purpose machine, for distinct $m$ and $n$, the finitely many argument places where $p_{i+m}$ fails to compute $f$ can be very different from the finitely many argument places where $p_{i+n}$ fails to compute $f$. One would hope though, that if Harrington's machine, or an improvement thereof, inferred the program $p_{i+m}$ based on the data points $f(0), f(1), \ldots, f(k)$, then $p_{i+m}$ would make very few mistakes computing $f$ at the "near future" arguments $k+1, k+2, \ldots, k+\ell$, where $\ell$ is reasonably large. Ideally, $p_{i+m}$ 's finitely many mistakes or anomalies would (mostly) occur at arguments $x \gg k$, i.e., ideally, its anomalies would be well placed beyond near future arguments. In the present paper, for general purpose learning machines, it is analyzed just how well or badly placed these anomalies may be with respect to near future arguments and what are the various tradeoffs.

In particular, there is good news and bad. Bad news is that, for any learning machine $\mathbf{M}$ (including general purpose $\mathbf{M}$ ), for all $m$, there exist infinitely many computable functions $f$ such that, infinitely often $\mathbf{M}$ incorrectly predicts $f$ 's next $m$

Preprint submitted to Elsevier Science 11 March 2007 
near future values. Good news is that, for a suitably clever general purpose learning machine $\mathbf{M}$, for each computable $f$, for $\mathbf{M}$ on $f$, the density of any such associated bad prediction intervals of size $m$ is vanishingly small.

Considered too is the possibility of providing a general purpose learner which additionally learns some interesting classes with respect to much stricter criteria than Bc*-identification. Again there is good news and bad. The criterion of $f_{-}$nite identification requires for success that a learner $\mathbf{M}$ on a function $f$ output exactly one program which correctly computes $f$. $\mathbf{B c}^{n}$-identification is just like Bc ${ }^{*}$-identification above except that the number of anomalies in each program of a final sequence is $\leq n$. Bad news is that there is a finitely identifiable class of computable functions $\mathcal{C}$ such that for no general purpose learner $\mathbf{M}$ and for no $n$, does $\mathbf{M}$ additionally $\mathbf{B} \mathbf{c}^{n}$-identify $\mathcal{C}$. Ex-identification by $\mathbf{M}$ on $f$ requires that $\mathbf{M}$ on $f$ converges, after a few output programs, to a single final program which computes $f$. A reliable learner (by definition) never deceives by false convergence; more precisely: whenever it converges to a final program on a function $f$, it must Ex-identify $f$. Good news is that, for any class $\mathcal{C}$ that can be reliably Ex-identified, there is a general purpose machine which additionally Ex-identifies $\mathcal{C}$ !

\section{Introduction}

The learning situation often studied in inductive inference [JORS99] may be described as follows. A learner receives as input, one at a time, the successive graph points of a function $f$. As the learner is receiving its input, it conjectures a sequence of programs as hypotheses. To be able to learn the function $f$, the sequence of programs conjectured by the learner must have some desirable relation to the input function $f$. By appropriately choosing this desirable relation one gets different criteria of successful learning. One of the first such criteria studied is called Ex-identification ([Gol67,BB75,CS83]). The learner is said to Ex-identify a function $f$ iff the sequence of programs output by it on $f$, after a few output programs, converges to a single final program which computes $f .{ }^{1}$ A learner is said to $\mathbf{E x}$-identify a class iff it $\mathbf{E x}$-identifies each function in the class. A class of functions is $\mathbf{E x}$-identifiable iff some machine Ex-identifies the class.

Even though one cannot Ex-identify the class of all the computable functions [Gol67], there are large and useful classes of functions which can be Ex-identified. For example, any recursively enumerable class of computable functions such as the class of polynomials or the class of primitive recursive functions [Rog67] is Ex-identifiable.

[Bār74,CS83] considered a generalization of Ex-identification called Bc-

1 In general more formal definitions are in Section 2 below. 
identification. In Bc-identification of a function $f$ by a machine $\mathbf{M}$ one requires that the sequence of programs output by $\mathbf{M}$ on $f$ either converges to a program for $f$, or the sequence of programs is infinite, with all but finitely many of them being (possibly different) programs for $f$. [CS83] also considered the variants of the $\mathbf{E x}$ and $\mathbf{B c}$-identification criteria in which the final programs need not be perfect, but are allowed to have some anomalies or mistakes in their predictions of $\mathrm{I} / \mathrm{O}$ behavior. For $n$ a natural number, if the final programs are allowed to make at most $n$ errors, then the criteria of inference are called $\mathbf{E} \mathbf{x}^{n}$ and $\mathbf{B} \mathbf{c}^{n}$ respectively. If the final programs are allowed to make at most finitely many errors, then the criteria of inference are called $\mathbf{E} \mathbf{x}^{*}$ and $\mathrm{Bc}^{*}$ respectively.

Harrington [CS83] constructed a machine which Bc ${ }^{*}$-identifies each computable function! In the present paper, we call machines which do this general purpose. However, on infinitely many computable functions, the final programs output by Harrington's machine become more and more degenerate, i.e., the finite sets of anomalies in successive final output programs, in general, grow in size without bound. We note that this is a property of any general purpose learner, and, in fact, the number of anomalies grows faster than any computable bound (Theorem 4 in Section 3 below).

Since the programs output by any general purpose learning machine make large numbers of mistakes (on infinitely many computable functions), it would be interesting to study how these errors are distributed. For example, in real life one probably cares more about "near future errors" than "distant future errors". Based on this motivation in Section 4 below we define new criteria of inference called $\mathbf{B} \mathbf{c}_{m}^{n}$. Informally, for a machine to $\mathbf{B} \mathbf{c}_{m}^{n}$-identify a function $f$, for its final programs, their predictions on the next $m$ inputs should have at most $n$ errors. In Section 4 we completely resolve the relationship between different $\mathbf{B c}_{m}^{n}$ criteria of inference (Corollary 23 in Section 4). In particular, we show that for any learning machine $\mathbf{M}$, (including general purpose $\mathbf{M}$ ), for all $m$, there exist infinitely many computable functions $f$ such that, infinitely often $\mathbf{M}$ incorrectly predicts $f$ 's next $m$ near future values (Corollary 24)! Thus there is an ostensibly unpleasant cost to general purpose learning. As we will see, though, this can, be assuaged at least in some interesting respects described below.

In contrast to the result mentioned above that any general purpose learning machine $\mathbf{M}$ predicts next $m$ values wrongly infinitely often, we show that the density of such bad prediction intervals can be made very small (Theorem 28 in Section 5 below).

A reliable learner (by definition) never deceives by false convergence; more precisely: whenever it converges to a final program on a function $f$, it must Ex-identify $f$ [Min76,BB75,CJNM94]. For example, r.e. classes of computable 
functions (such as the class of polynomial functions and the class of primitive recursive functions [Rog67]) as well as the class of total run time functions can be reliably Ex-identified [BB75,CS83]. On a further positive note, we show that for every reliably Ex-identifiable class of computable functions $\mathcal{S}$, there is a general purpose learning machine which Ex-identifies $\mathcal{S}$ (Theorem 30 in Section 5 below)!

The criterion of finite identification requires for success that a learner $\mathbf{M}$ on a function $f$ output exactly one program which correctly computes $f$. Learning by finite identification can be thought of as one-shot learning. We show, by contrast to the result in the immediately above paragraph (Theorem 30), that there is a class $\mathcal{S}$ which is finitely identifiable, yet for all $n$, no general purpose learner can additionally $\mathbf{B c}^{n}$-identify $\mathcal{S}$ (Corollary 36 in Section 5 below).

Freivalds and Wiehagen [FW79] showed that there exists a machine which can identify all the recursive functions if, in addition to the graph of the input function, it is given an arbitrary upper bound on the size of the minimal program computing the input function as additional information. Freivalds, Botuscharov and Wiehagen [FBW98] further showed that in some (but not all) acceptable programming systems, the above machine can produce a final program of size within the upper bound given as additional information. Machines, as in above, exhibit a different kind of general purpose behaviour. We will not deal with above type of general purpose learners in this paper.

We now proceed formally.

\section{Notation and Preliminaries}

Recursion-theoretic concepts not explained below are treated in [Rog67]. $N$ denotes the set of natural numbers. $*$ denotes a non-member of $N$ and is assumed to satisfy $(\forall n \in N)[n<*<\infty]$. Let $\in, \subseteq, \subset, \supseteq, \supset$, respectively denote membership, subset, proper subset, superset and proper superset relations for sets. Emptyset is denoted by $\emptyset$. Cardinality of a set $S$ is denoted by $\operatorname{card}(S)$. So "card $(S) \leq *$ " means that $\operatorname{card}(S)$ is finite. We let $\min (S)$ and $\max (S)$, respectively, denote the minimum and maximum element in $S$. We take $\min (\emptyset)$ to be $\infty$ and $\max (\emptyset)$ to be 0 .

$\langle\cdot, \cdot\rangle$ denotes a 1-1 computable mapping from pairs of natural numbers onto natural numbers. $\pi_{1}, \pi_{2}$ are the corresponding projection functions. $\langle\cdot, \cdot\rangle$ is extended to $n$-tuples in a natural way.

$\Lambda$ denotes the empty function. $\eta$, with or without decorations (decorations are subscripts, superscripts, primes and such), ranges over partial functions. 
$\eta(x) \downarrow$ denotes that $\eta(x)$ is defined. $\eta(x) \uparrow$ denotes that $\eta(x)$ is not defined. For $a \in N \cup\{*\}, \eta_{1}=^{a} \eta_{2}$ means that $\operatorname{card}\left(\left\{x \mid \eta_{1}(x) \neq \eta_{2}(x)\right\}\right) \leq a \cdot \eta_{1} \neq^{a} \eta_{2}$ means that $\neg\left[\eta_{1}={ }^{a} \eta_{2}\right]$. (If $\eta_{1}$ and $\eta_{2}$ are both undefined on input $x$, then, as is standard, we take $\eta_{1}(x)=\eta_{2}(x)$.) If $\eta={ }^{a} f$, then we often call a program for $\eta$ as an $a$-error program for $f$. We let $\operatorname{domain}(\eta)$ and range $(\eta)$ respectively denote the domain and range of the partial function $\eta$.

We let $f, g$ and $h$, with or without decorations, range over total functions. $\mathcal{R}$ denotes the class of all computable functions, i.e., total computable functions with arguments and values from $N . \mathcal{C}$ and $\mathcal{S}$, with or without decorations, range over subsets of $\mathcal{R}$. $\varphi$ denotes a fixed acceptable programming system $[\operatorname{Rog} 58, \operatorname{Rog} 67, \operatorname{Ric} 80, \operatorname{Ric} 81, \operatorname{Roy} 87] . \varphi_{i}$ denotes the partial computable function computed by program $i$ in the $\varphi$-system. Note that in this paper all programs are interpreted with respect to the $\varphi$-system. We let $\Phi$ be an arbitrary Blum complexity measure [Blu67] associated with the acceptable programming system $\varphi$; many such measures exist for any acceptable programming system [Blu67]. Let $\varphi_{i, s}$ be defined as follows.

$$
\varphi_{i, s}(x)= \begin{cases}\varphi_{i}(x), & \text { if } x<s \text { and } \Phi_{i}(x) \leq s \\ \uparrow, & \text { otherwise. }\end{cases}
$$

For a given partial computable function $\eta$, we define $\operatorname{MinProg}(\eta)$ to denote $\min \left(\left\{i \mid \varphi_{i}=\eta\right\}\right)$.

Let zeroext $(\eta)$ denote a function defined as follows.

$$
\operatorname{zeroext}(\eta)(x)= \begin{cases}\eta(x), & \text { if } x \in \operatorname{domain}(\eta) \\ 0, & \text { otherwise }\end{cases}
$$

\subsection{Function Identification}

We first describe inductive inference machines. We assume, without loss of generality, that the graph of a function is fed to a machine in canonical order. For any partial function $\eta$ and $n \in N$ such that, for all $x<n, \eta(x) \downarrow$, we let $\eta[n]$ denote the finite initial segment $\{(x, \eta(x)) \mid x<n\}$. Clearly, $\eta[0]$ denotes the empty segment. We let $\Lambda$ denote the empty segment. SEG denotes the set of all finite initial segments, $\{f[n] \mid f \in \mathcal{R} \wedge n \in N\}$. We let $\sigma$ and $\tau$, with or without decorations, range over SEG. Let $|\sigma|$ denote the length of $\sigma$. We often identify (partial) functions with their graphs. Thus for example, for $\sigma=f[n]$ and for $x<n, \sigma(x)$ denotes $f(x)$. A learning machine (also called an inductive inference machine (IIM)) [Gol67] is an algorithmic device that computes a mapping from SEG into $N \cup\{$ ?\}. Intuitively, "?" above denotes the case when the machine may not wish to make a conjecture. Although it 
is not necessary to consider learners that issue "?" for identification in the limit, it becomes useful when the number of mind changes a learner can make is bounded. In this paper, we assume, without loss of generality, that once an IIM has issued a conjecture on some initial segment of a function, it outputs a conjecture on all extensions of that initial segment. This is without loss of generality because a machine wishing to emit "?" after making a conjecture can instead be thought of as repeating its previous conjecture. We let $\mathbf{M}$, with or without decorations, range over learning machines. Since the set of all finite initial segments, SEG, can be coded onto $N$, we can view these machines as taking natural numbers as input and emitting natural numbers or ?'s as output. We say that $\mathbf{M}(f)$ converges to $i$ (written: $\mathbf{M}(f) \downarrow=i$ ) iff $\left(\forall^{\infty} n\right)[\mathbf{M}(f[n])=i] ; \mathbf{M}(f)$ is undefined if no such $i$ exists. The next definitions describe several criteria of function identification.

Definition 1 [Gol67,BB75,CS83] Let $a \in N \cup\{*\}$. Let $f \in \mathcal{R}$.

(a) $\mathbf{M} \mathbf{E} \mathbf{x}^{a}$-identifies $f$ (written: $f \in \mathbf{E x}^{a}(\mathbf{M})$ ) just in case, there exists an $i$ such that $\mathbf{M}(f) \downarrow=i$ and $\varphi_{i}={ }^{a} f$.

(b) $\mathbf{M} \mathbf{E x}{ }^{a}$-identifies $\mathcal{S}$ iff $\mathbf{M} \mathbf{E x}{ }^{a}$-identifies each $f \in \mathcal{S}$.

(c) $\mathbf{E x}^{a}=\left\{\mathcal{S} \subseteq \mathcal{R} \mid(\exists \mathbf{M})\left[\mathcal{S} \subseteq \mathbf{E x}^{a}(\mathbf{M})\right]\right\}$.

We often write $\mathbf{E x}$ for $\mathbf{E x}^{0}$.

By definition of convergence, only finitely many data points from a function $f$ had been observed by an IIM $\mathbf{M}$ at the (unknown) point of convergence. Hence, some form of learning must take place in order for $\mathbf{M}$ to learn $f$. For this reason, hereafter the terms identify, learn and infer are used interchangeably.

Definition 2 [Bār74,CS83] Let $a \in N \cup\{*\}$. Let $f \in \mathcal{R}$.

(a) $\mathbf{M} \mathbf{B} \mathbf{c}^{a}$-identifies $f$ (written: $f \in \mathbf{B c}^{a}(\mathbf{M})$ ) iff, for all but finitely many $n \in N, \varphi_{\mathbf{M}(f[n])}=^{a} f$.

(b) $\mathbf{M} \mathbf{B c}^{a}$-identifies $\mathcal{S}$ iff $\mathbf{M} \mathbf{B c}^{a}$-identifies each $f \in \mathcal{S}$.

(c) $\mathrm{Bc}^{a}=\left\{\mathcal{S} \subseteq \mathcal{R} \mid(\exists \mathbf{M})\left[\mathcal{S} \subseteq \mathrm{Bc}^{a}(\mathbf{M})\right]\right\}$.

We often write $\mathbf{B c}$ for $\mathbf{B c}^{0}$.

Some relationships between the above criteria are summarized in the following theorem.

Theorem 3 [CS83,BB75,Bār71]

$\mathbf{E x}^{0} \subset \mathbf{E x}^{1} \subset \cdots \subset \mathbf{E x}^{*} \subset \mathbf{B c} \subset \mathbf{B c}^{1} \subset \cdots \subset \mathbf{B c}^{*}=2^{\mathcal{R}}$. 
Since $\mathcal{R} \in \mathbf{B c}^{*}$, we often call a machine which $\mathbf{B} \mathbf{c}^{*}$-identifies $\mathcal{R}$ a general purpose learning machine.

We let $\mathbf{I}$ range over identification criteria defined above. There exists an r.e. sequence $\mathbf{M}_{0}, \mathbf{M}_{1}, \mathbf{M}_{2}, \ldots$, of inductive inference machines such that, for all criteria I of inference considered in this paper, one can show that [JORS99]:

for all $\mathcal{C} \in \mathbf{I}$, there exists an $i \in N$ such that $\mathcal{C} \subseteq \mathbf{I}\left(\mathbf{M}_{i}\right)$.

We assume $\mathbf{M}_{0}, \mathbf{M}_{1}, \mathbf{M}_{2}, \ldots$ to be one such sequence of machines.

\section{General Purpose Machines and Their Mistakes}

Unfortunately, the programs output by Harrington's machine become more and more degenerate, i.e. the finite set of anomalies in final programs output grows in size without bound. In fact the finite sets of anomalies cannot even be bounded by a computable function as the next theorem shows.

Theorem 4 Suppose $\mathcal{R} \subseteq \mathbf{B c}^{*}(\mathbf{M})$. Let $g$ be a computable function. Then there exist infinitely many $f \in \mathcal{R}$ such that, for infinitely many $n, \varphi_{\mathbf{M}(f[n])} \neq^{g(n)}$ $f$.

Proof. Suppose $\mathbf{M}$ and $g$ are as given in the hypothesis. We will construct one $f$ such that for infinitely many $n, \varphi_{\mathbf{M}(f[n])} \neq^{g(n)} f$. The construction can be easily modified to produce infinitely many distinct such $f$. By Kleene Recursion Theorem [Rog67], there exists an $e$ such that $\varphi_{e}$ may be defined as follows. Let $\varphi_{e}(0)=e$. Let $x_{0}=1$. Go to stage 0 .

Stage $s$

1. Let $h=\operatorname{zeroext}\left(\varphi_{e}\left[x_{s}\right]\right)$.

2. Search for $n_{s}>x_{s}$ and a set $S_{s}$ such that (a) $\varphi_{\mathbf{M}\left(h\left[n_{s}\right]\right)}(y) \downarrow$, for all $y \in S_{s}$, (b) $\min \left(S_{s}\right)>n_{s}$, and (c) $\operatorname{card}\left(S_{s}\right)>g\left(n_{s}\right)$.

3. If and when such $n_{s}, S_{s}$ are found, let $\varphi_{e}(y)=1+\varphi_{\mathbf{M}\left(h\left[n_{s}\right]\right)}(y)$, if $y \in S_{s}$. $\varphi_{e}(y)=h(y)$, if $y \leq \max \left(S_{s}\right)$ and $y \notin S_{s}$.

4. Let $x_{s+1}=1+\max \left(S_{s}\right)$.

Go to stage $s+1$.

End stage $s$

We first claim that step 2 succeeds in every stage. This is so since $\mathbf{M} \mathbf{B} \mathbf{c}^{*}$ identifies all computable functions, and in particular $h$. Thus, there exist $n_{s}$ and $S_{s}$ such that (a) $\varphi_{\mathbf{M}\left(h\left[n_{s}\right]\right)}(y) \downarrow$, for all $y \in S_{s}$, (b) $\min \left(S_{s}\right)>n_{s}$, and 
(c) $\operatorname{card}\left(S_{s}\right)>g\left(n_{s}\right)$. It follows that $\varphi_{e}$ is total. Let $f=\varphi_{e}$. Now for all $s, \varphi_{\mathbf{M}\left(f\left[n_{s}\right]\right)}(x) \neq f(x)$, for all $x \in S_{s}$. Thus, $\varphi_{\mathbf{M}\left(f\left[n_{s}\right]\right)} \neq^{g\left(n_{s}\right)} f$. Theorem follows.

\section{Predicting Near Future Values}

Based on Theorem 4, it would be interesting to study how the anomalies of the programs outputted by a machine are distributed. For example, in real life one probably cares more about "near future errors" than "distant future errors". This leads us to the following definition.

Definition 5 Let $m, n \in N$ and $f \in \mathcal{R}$.

(a) $\mathbf{M} \mathbf{B} \mathbf{c}_{m}^{n}$-identifies $f$ (written: $f \in \mathbf{B c}_{m}^{n}(\mathbf{M})$ ), iff, for all but finitely many $x, \operatorname{card}\left(\left\{z<m \mid \varphi_{\mathbf{M}(f[x])}(x+z) \neq f(x+z)\right\}\right) \leq n$.

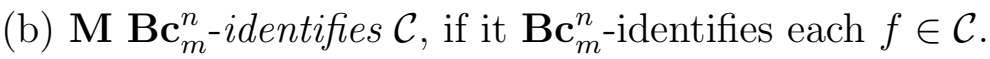

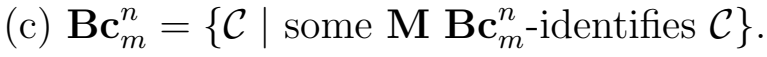

Intuitively, one can view $\mathbf{B c}_{m}^{n}$-identification of a function by a machine as follows. At any stage, the learning machine predicts the next $m$ values. At all but finitely many stages, at least $m-n$ out of the $m$ predictions are correct.

In this section we resolve the relationship between different $\mathbf{B c}_{m}^{n}$-identification criteria.

Following four propositions follow directly from the definitions.

Proposition 6 For $m \geq n, \mathbf{B c}^{n} \subseteq \mathbf{B c}_{m}^{n}$.

Proposition 7 Suppose $m \geq n$ and $k \in N$. Then $\mathbf{B c}_{m}^{n} \subseteq \mathbf{B c}_{m+k}^{n+k}$.

Proposition 8 Suppose $m \geq n \geq k$. Then $\mathbf{B c}_{m}^{k} \subseteq \mathbf{B} \mathbf{c}_{m}^{n}$.

Proposition 9 Suppose $m \geq k \geq n$. Then $\mathbf{B c}_{m}^{n} \subseteq \mathbf{B} \mathbf{c}_{k}^{n}$.

Proposition 10 For all $m>0, \mathbf{B c}=\mathbf{B c}_{m}^{0}$.

Proof. Clearly, for $m \geq 1$, Bc $\subseteq \mathbf{B c}_{m}^{0} \subseteq \mathbf{B c}_{1}^{0}$. NV" defined by Podnieks $[\mathrm{Pod} 74]$ is identical to $\mathbf{B c}_{1}^{0}$. The proposition follows from $\mathbf{N V}^{\prime \prime}=\mathbf{B c}$ [Pod74,CS83].

The following theorem shows some advantages of having to predict fewer correct values in the near future. 
Theorem 11 Suppose $m^{\prime}>m$. Then, $\mathbf{B c}_{m}^{1}-\mathbf{B c}_{m^{\prime}}^{m^{\prime}-m} \neq \emptyset$.

Proof. Let

$$
\begin{gathered}
Z_{k}=\left\{x \mid k \cdot m^{\prime}<x<k \cdot m^{\prime}+m\right\}, \\
E_{k}=\left\{k \cdot m^{\prime}\right\} \cup\left\{x \mid k \cdot m^{\prime}+m \leq x<(k+1) \cdot m^{\prime}\right\},
\end{gathered}
$$

and

$$
U_{k}=Z_{k} \cup E_{k}=\left\{x \mid k \cdot m^{\prime} \leq x<(k+1) \cdot m^{\prime}\right\} .
$$

We now consider the following two properties defined on total functions.

(PropA) $f$ satisfies PropA iff, for all $k$, for all $x \in Z_{k}, f(x)=0$.

(PropB) $f$ satisfies PropB iff, for all $k$, for all $x \in E_{k}, f(x)=f\left(k \cdot m^{\prime}\right)$.

Let $\mathcal{C}=\{f \in \mathcal{R} \mid f$ satisfies PropA and PropB $\}$.

The above class is easily seen to be in $\mathbf{B} \mathbf{c}_{m}^{1}$. We now show that $\mathcal{C} \notin \mathbf{B c}_{m^{\prime}}^{m^{\prime}-m}$.

Suppose by way of contradiction that $\mathbf{M} \mathbf{B c}_{m^{\prime}}^{m^{\prime}-m}$-identifies $\mathcal{C}$. Let $\sigma_{0}=\Lambda$. In the following construction, in stage $s$ we will define $\sigma_{s+1}$. Domain of $\sigma_{s}$ will be $\bigcup_{k<s} U_{k}$. Also, $\lim _{s \rightarrow \infty} \sigma_{s}(x) \downarrow$. Suppose $f$ is defined as follows:

$$
f(x)=\lim _{s \rightarrow \infty} \sigma_{s}(x)
$$

Then, $f$ will be in $\mathcal{C}$, and $\mathbf{M}$ does not $\mathbf{B c}_{m^{\prime}}^{m^{\prime}-m}$-identifies $f$.

For all $k$, let $\operatorname{err}_{0}^{k}=0$. Intuitively, at the beginning of stage $s$, for $k<s$, $\operatorname{err}_{s}^{k}$ denotes the number of inputs in $E_{k}$ on which $\mathbf{M}\left(\sigma_{s}\left[k \cdot m^{\prime}\right]\right)$ has been currently diagonalized against. We will have $\operatorname{err}_{s}^{k}=0$, for $k \geq s$.

Go to stage 0 .

Stage $s$

1. For $k \leq s$, let $\operatorname{Conv}_{s}^{k}=\operatorname{card}\left(\left\{x \in E_{k} \mid \varphi_{\mathbf{M}\left(\sigma_{s}\left[k \cdot m^{\prime}\right]\right), s}(x) \downarrow\right\}\right)$.

2. Let $\operatorname{Cand}_{s}=\left\{k \leq s \mid \operatorname{Conv}_{s}^{k}>\max \left(\left\{\operatorname{err}_{s}^{k^{\prime}} \mid k \leq k^{\prime} \leq s\right\}\right)\right\}$.

If Cand $\cot _{s}=\emptyset$, then let $C_{s}=s$. Otherwise, let $C_{s}$ be the minimum element in $\mathrm{Cand}_{s}$.

3. For $k<C_{s}$, let $\operatorname{err}_{s+1}^{k}=\operatorname{err}_{s}^{k}$.

Let $\operatorname{err}_{s+1}^{C_{s}}=\operatorname{Conv}_{s}^{C_{s}}$.

For $k>C_{s}$, let $\mathrm{err}_{s+1}^{k}=0$.

4. For $x \in \bigcup_{k<C_{s}} U_{k}$, let $\sigma_{s+1}(x)=\sigma_{s}(x)$.

For $x \in E_{C_{s}}$, let $\sigma_{s+1}(x)=1+\max \left(\left\{\varphi_{\mathbf{M}\left(\sigma_{s}\left[C_{s} \cdot m^{\prime}\right]\right)}(x) \mid x \in\right.\right.$ $\left.\left.E_{C_{s}} \wedge \varphi_{\mathbf{M}\left(\sigma_{s}\left[C_{s} \cdot m^{\prime}\right]\right), s}(x) \downarrow\right\}\right)$.

For $x \in Z_{C_{s}} \cup \cup_{C_{s}<k<s+1} U_{k}$, let $\sigma_{s+1}(x)=0$.

5. Go to stage $s+1$.

End stage $s$ 
Claim 12 For all s, the following three properties hold.

(a) for all $x \in \bigcup_{k<C_{s}} U_{k}, \sigma_{s+1}(x)=\sigma_{s}(x)$.

(b) for all $k<C_{s}$, $\operatorname{err}_{s+1}^{k}=\operatorname{err}_{s}^{k}$, and

(c) either $C_{s}=s$, or $\operatorname{err}_{s+1}^{C_{s}}>\max \left(\left\{\operatorname{err}_{s}^{k} \mid C_{s} \leq k \leq s\right\}\right)$.

Proof. Follows directly from construction. $\square$ (Claim 12)

Claim $13 \lim _{s \rightarrow \infty} \operatorname{err}_{s}^{k} \downarrow$ for every $k$. Thus, $\liminf C_{s}$ goes to infinity, and $\lim _{s \rightarrow \infty} \sigma_{s}(x) \downarrow$.

\section{ProOF.}

We show the claim by induction on $k$. Suppose, for $k^{\prime}<k, \lim _{s \rightarrow \infty} \operatorname{err}_{s}^{k^{\prime}} \downarrow$. Let $t>k$ be a stage such that, for all $k^{\prime}<k$, for all $s>t$, $\operatorname{err}_{s}^{k^{\prime}}=\operatorname{err}_{t}^{k^{\prime}}$. Thus, by Claim 12(b and c), for all $s>t, C_{s} \geq k$. Hence, by Claim 12(b and c), for all $s>t$, $\operatorname{err}_{s}^{k} \leq \operatorname{err}_{s+1}^{k}$. Since $\operatorname{err}_{s}^{k}$ is bounded by $m^{\prime}-m+1$, $\lim _{s \rightarrow \infty} \operatorname{err}_{s}^{k} \downarrow$. Thus, using Claim 12, it follows that $\lim \inf C_{s}$ goes to infinity, and thus $\lim _{s \rightarrow \infty} \sigma_{s}(x) \downarrow . \square($ Claim 13)

Let

$$
f(x)=\lim _{s \rightarrow \infty} \sigma_{s}(x)
$$

We claim that $f \in \mathcal{C}$ and $\mathbf{M}$ does not $\mathbf{B c}_{m^{\prime}}^{m^{\prime}-m}$-identify $f$. Note that $f$ clearly satisfies PropA and PropB. We thus just need to show that $f \in \mathcal{R}$ and $\mathbf{M}$ does not $\mathbf{B c}_{m^{\prime}}^{m^{\prime}-m}$-identify $f$.

Let $\operatorname{err}^{k}$ denote $\lim _{s \rightarrow \infty} \operatorname{err}_{s}^{k}$. Let $r \leq m^{\prime}-m+1$, be the largest value such that $\mathrm{err}^{k}=r$, for infinitely many $k$.

Let $t_{1}, t_{2}$ be large enough so that, $t_{2}>t_{1}$, and

(i) for all $k \geq t_{1}$, err $^{k} \leq r$;

(ii) for all $k \leq t_{1}$, for all $s \geq t_{2}$, $\operatorname{err}_{s}^{k}=\operatorname{err}_{t_{2}}^{k}$.

Thus, in particular for all $s>t_{2}, \operatorname{err}_{s}^{C_{s}} \leq r$.

Claim 14 For all $s>t_{2}$, if $\operatorname{err}_{s}^{C_{s}}=r$, then for all $s^{\prime}>s, C_{s^{\prime}}>C_{s}$,

Proof. Suppose by way of contradiction that $s^{\prime}>s$ is the first stage in which $C_{s^{\prime}} \leq C_{s}$. Then $\operatorname{err}_{s^{\prime}}^{C_{s^{\prime}}}>\operatorname{err}_{s}^{C_{s}}$, by the condition for selection of $C_{s^{\prime}}$ in step 2 of the construction. This in turn implies that $\operatorname{err}_{s^{\prime}}^{C_{s^{\prime}}}>r$. A contradiction to the choice of $t_{2} . \square$ (Claim 14) 
It follows from Claims 12 and 14 that, if $s>k_{2}$ and $\operatorname{err}_{s}^{C_{s}}=r$, then $\sigma_{s+1}\left[\left(C_{s}+\right.\right.$ $\left.1) \cdot m^{\prime}\right] \subseteq f$. Moreover, since $\operatorname{err}_{s}^{C_{s}}=r$, for infinitely many $s$, it follows that

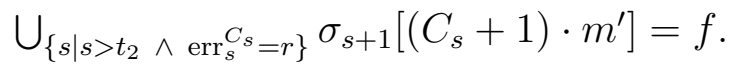

Since the left hand side of above equation is computable, it follows that $f$ is computable.

Furthermore, for all $s>t_{2}$, for all $k>t_{1}$, Conv ${ }_{s}^{k} \leq r$ (otherwise $\operatorname{err}_{s}^{C_{s}}$ would be at least $r+1$ ).

Thus, for all $C_{s}>t_{1}$, such that $\operatorname{err}_{s}^{C_{s}}=r$, we have $\varphi_{\mathbf{M}\left(f\left[C_{s} \cdot m^{\prime}\right]\right)}(x) \neq f(x)$, for all $x \in E_{C_{s}}$. It follows that $\mathbf{M}$ does not $\mathbf{B c}_{m^{\prime}}^{m^{\prime}-m}$-identify $f$. Theorem follows. $\square$ (Theorem 11)

The next theorem shows some advantages of being allowed to predict more wrong values in the near future.

Theorem 15 For all $n \in N, \mathbf{B c}^{n+1}-\mathbf{B c}_{n+1}^{n} \neq \emptyset$.

Proof. Let $Z_{f}=\{x \mid f((n+2) \cdot x) \neq 0\}$.

Let $\mathcal{C}=\left\{f \mid\left[\operatorname{card}\left(Z_{f}\right)=\infty \wedge\left(\forall^{\infty} x \in Z_{f}\right)\left[\varphi_{f((n+2) \cdot x)}=^{n+1} f\right]\right] \vee[0<\right.$ $\left.\left.\operatorname{card}\left(Z_{f}\right)<\infty \wedge \varphi_{f\left((n+2) \cdot \max \left(Z_{f}\right)\right)}=^{n+1} f\right]\right\}$.

It is easy to verify that $\mathcal{C} \in \mathbf{B c}^{n+1}$. We now show that $\mathcal{C} \notin \mathbf{B c}_{n+1}^{n}$. Suppose by way of contradiction, $\mathbf{M} \mathbf{B c}_{n+1}^{n}$-identifies $\mathcal{C}$. Then, by operator recursion theorem [Cas74], there exists a computable, $1-1$, increasing $p$ such that $p(0)>$ 0 , and $\varphi_{p(i)}$ may be described in stages as follows.

Below, let $\varphi_{p(y)}^{s}$ denote $\varphi_{p(y)}$ defined before stage $s$. Let $X_{k}=\{x \mid(n+2) \cdot k<$ $x<(n+2) \cdot(k+1)\}$. Intuitively, for the diagonalizing function $f$ constructed, for infinitely many $k$, for all $x \in X_{k}, \varphi_{\mathbf{M}(f[(n+2) \cdot k+1])}(x) \neq f(x)$.

We will define variables $q_{s}^{i}$ (for $\left.i \leq n+2\right), \sigma_{s}$, and $E_{s}^{i}($ for $i \leq n+1)$ in the construction. Intuitively, for the diagonalizing function $f$ which we will construct, think of $q_{s}^{i}$ (for $i \leq n+1$ ) as elements of $Z_{f} . \sigma_{s}$ is an approximate initial segment of $f$ at the beginning of stage $s$. The domain of $\sigma_{s}$ will be $\left\{x \mid x<(n+2) \cdot\left(1+q_{s}^{n+2}\right)\right\} . E_{s}^{i}$ denotes a set of $k$ 's such that $q_{s}^{i}<k \leq q_{s}^{i+1}$ and $\varphi_{\mathbf{M}\left(\sigma_{s}[(n+2) \cdot k+1]\right)}$ makes at least $n+1-i$ convergent errors on inputs from $X_{k}$ (with respect to $\sigma_{s}$ ). 
For $i \leq n+2$, let $q_{0}^{i}=i$. Initial segment $\sigma_{0}$, with domain $\{x \mid x<(n+2)$. $\left.\left(q_{0}^{n+2}+1\right)\right\}$ is defined as follows.

$$
\sigma_{0}(x)= \begin{cases}p\left(q_{0}^{i}\right), & \text { if } x=q_{0}^{i} \cdot(n+2), \text { for some } i<n+2 \\ 0, & \text { if } x<(n+2) \cdot\left(q_{0}^{n+2}+1\right) \text { and } x \text { is not of } \\ & \text { form } q_{0}^{i} \cdot(n+2), \text { for any } i<n+2 .\end{cases}
$$

For $i \leq n+1$, let $\varphi_{p\left(q_{s}^{i}\right)}^{0}=\sigma_{s}\left[(n+2) \cdot\left(1+q_{s}^{i+1}\right)\right]$.

For $i<n+1$, let $E_{0}^{i}=\emptyset$, and let $E_{0}^{n+1}=\left\{q_{s}^{n+2}\right\}$. In stage $s$, we will define $\sigma_{s+1}$ and $q_{s+1}^{i}$, for $i \leq n+2$, and correspondingly define $E_{s+1}^{i}$, for $i \leq n+1$. This will be done is such a way, so that (a) $(\forall x)\left(\forall^{\infty} s\right)\left[\sigma_{s+1}(x)=\sigma_{s}(x)\right]$, and (b) $f$ defined as $f(x)=\lim _{s \rightarrow \infty} \sigma_{s}(x)$, is the diagonalizing function.

For all $s$, we will satisfy the following invariants:

(A) For $i \leq n, q_{s}^{i}<q_{s}^{i+1}$.

(B) For all $i \leq n+1, \varphi_{p\left(q_{s}^{i}\right)}^{s}=\sigma_{s}\left[(n+2) \cdot\left(q_{s}^{i+1}+1\right)\right]$.

(C) For all $i \leq n+1$, for all $k \in E_{s}^{i}, \operatorname{card}\left(\left\{x \in X_{k} \mid \varphi_{\mathrm{M}\left(\sigma_{s}[(n+2) \cdot k+1]\right)}^{s}(x) \downarrow \neq\right.\right.$ $\left.\left.\sigma_{s}(x)\right\}\right) \geq n+1-i$.

(D) For all $i \leq n+1, E_{s}^{i} \subseteq\left\{x \mid q_{s}^{i}<k \leq q_{s}^{i+1}\right\}$.

Go to stage 0 .

Stage $s$

1. Let $\operatorname{Cand}_{s}=\left\{i<n+1 \mid\left(\exists k \in E_{s}^{i+1}\right)\left[\operatorname{card}\left(\left\{x \in X_{k} \mid\right.\right.\right.\right.$ $\left.\left.\left.\left.\varphi_{\mathbf{M}\left(\sigma_{s}[k \cdot(n+2)+1]\right), s}(x) \downarrow\right\}\right) \geq n+1-i\right]\right\}$.

2. If $\operatorname{Cand}_{s}$ is empty, then let $C_{s}=n+1$. Else, let $C_{s}=\min \left(\operatorname{Cand}_{s}\right)$.

3. If $C_{s}<n+1$, then let $k_{s} \in E_{s}^{C_{s}+1}$ be such that $\operatorname{card}\left(\left\{x \in X_{k_{s}} \mid\right.\right.$ $\left.\left.\varphi_{\mathbf{M}\left(\sigma_{s}\left[k_{s} \cdot(n+2)+1\right]\right), s}(x) \downarrow\right\}\right) \geq n+1-i$.

If $C_{s}<n+1$ and there exists an $x \in X_{k_{s}}$ such that $\sigma_{s}(x)=$ $\varphi_{\mathbf{M}\left(\sigma_{s}\left[k_{s} \cdot(n+2)+1\right]\right), s}\left(y_{s}\right) \downarrow$, then let $y_{s}$ be one such $x$. Else, let $y_{s}=\uparrow$.

4. For $i \leq C_{s}$, let $q_{s+1}^{i}=q_{s}^{i}$.

For $C_{s}<i \leq n+2$, let $q_{s+1}^{i}=q_{s}^{n+2}+i-C_{s}$. 
5. Let

$$
\sigma_{s+1}(x)= \begin{cases}\sigma_{s}(x), & \text { if } x<(n+2) \cdot\left(1+q_{s}^{n+2}\right) \\ \varphi_{\mathbf{M}\left(\sigma_{s}\left[k_{s} \cdot(n+2)+1\right]\right), s}\left(y_{s}\right)+1, & \text { if } x=y_{s} ; \\ p\left(q_{s+1}^{i}\right), & \text { if }(n+2) \cdot\left(1+q_{s}^{n+2}\right) \leq x \\ & \text { and } x<(n+2) \cdot\left(1+q_{s+1}^{n+2}\right) \\ & \text { and } x=q_{s+1}^{i} \cdot(n+2), \\ & \text { for some } i<n+2 ; \\ & \text { if }(n+2) \cdot\left(1+q_{s}^{n+2}\right) \leq x \\ & \text { and } x<(n+2) \cdot\left(1+q_{s+1}^{n+2}\right) \\ & \text { and } x \text { is not of form } \\ & q_{s+1}^{i} \cdot(n+2), \\ & \text { for any } i<n+2 .\end{cases}
$$

6. For $i<C_{s}$, let $E_{s+1}^{i}=E_{s}^{i}$.

If $C_{s}<n+1$, then let $E_{s+1}^{C_{s}}=E_{s}^{C_{s}} \cup\left\{k_{s}\right\}$.

For $C_{s}<i<n+1$, let $E_{s+1}^{i}=\emptyset$.

Let $E_{s+1}^{n+1}=\left\{k \mid q_{s+1}^{n+1}<k \leq q_{s+1}^{n+2}\right\}$.

7. For $i \leq n+1$, let $\varphi_{p\left(q_{s+1}^{i}\right)}^{s+1}=\sigma_{s+1}\left[(n+2) \cdot\left(q_{s+1}^{i+1}+1\right)\right]$.

8. For $C_{s}<i \leq n+1$, let $\varphi_{p\left(q_{s}^{i}\right)}$ follow $\varphi_{p\left(q_{s+1}^{C_{s}}\right)}$ on inputs $\geq(n+2) \cdot\left(q_{s}^{i+1}+1\right)$.

(* Note that above implies that $\varphi_{p\left(q_{s}^{i}\right)}={ }^{1} \varphi_{p\left(q_{s+1}^{C_{s}}\right)}$, since $\varphi_{p\left(q_{s}^{i}\right)}$ and $\varphi_{p\left(q_{s+1}^{C_{s}}\right)}$ may differ only on $y_{s}{ }^{*}$ )

9. Go to stage $s+1$.

End stage $s$

It is easy to verify that invariants (A) to (D) are satisfied. Also, note that step 7 is consistent since, for $i \leq C_{s}, \sigma_{s}\left[(n+2) \cdot\left(q_{s}^{i+1}+1\right)\right] \subseteq \sigma_{s+1}\left[(n+2) \cdot\left(q_{s+1}^{i+1}+1\right)\right]$ (by invariant (D), if $C_{s}<n+1$, then $k_{s}>q_{s}^{C_{s}+1}$ and thus $\left.y_{s}>(n+2) \cdot\left(q_{s}^{C_{s}+1}+1\right)\right)$.

Claim 16 For each s, the following are satisfied.

(a) For $i \leq C_{s}, q_{s+1}^{i}=q_{s}^{i}$.

(b) For $C_{s}<i \leq n+2, q_{s+1}^{i}>q_{s}^{n+2}$.

(c) $q_{s}^{i} \leq q_{s+1}^{i}$.

(d) For $i<j$, if $q_{s}^{i}<q_{s^{\prime}}^{j}$, then $s \leq s^{\prime}$ or $q_{s}^{i}=q_{s^{\prime}}^{i}$.

(e) $\sigma_{s}\left[(n+2) \cdot\left(q_{s}^{C_{s}+1}+1\right)\right] \subseteq \sigma_{s+1}\left[(n+2) \cdot\left(q_{s}^{C_{s}+1}+1\right)\right]$.

(f) For all $C_{s}<i \leq n+1, \varphi_{p\left(q_{s}^{i}\right)}={ }^{1} \varphi_{p\left(q_{s}^{C_{s}}\right)}$. 
Proof. Parts (a) to (e) follow by induction on stages. For (f) see note in step 8. $\square$ (Claim 16)

Claim 17 For all $s$ and for all $i<n+1, \operatorname{card}\left(\left\{x \mid \varphi_{p\left(q_{s}^{i}\right)}(x) \downarrow \neq \varphi_{p\left(q_{s}^{i+1}\right)}(x)\right\}\right) \leq$ 1.

Proof. If for all $s^{\prime} \geq s, q_{s^{\prime}}^{i+1}=q_{s}^{i+1}$, then clearly, $\varphi_{p\left(q_{s}^{i}\right)}=\varphi_{p\left(q_{s}^{i}\right)}^{s}=\sigma_{s}[(n+$ $\left.2) \cdot\left(q_{s}^{i+1}+1\right)\right] \subseteq \varphi_{p\left(q_{s}^{i+1}\right)}^{s} \subseteq \varphi_{p\left(q_{s}^{i+1}\right)}$.

So suppose $s^{\prime}>s$ is the least number such that $q_{s^{\prime}}^{i+1} \neq q_{s}^{i+1}$. Then, by construction of $\sigma_{s^{\prime}}$ and steps 7 and 8 in stage $s^{\prime}-1$, the only possible member of $\left\{x \mid \varphi_{p\left(q_{s}^{i}\right)}(x) \downarrow \neq \varphi_{p\left(q_{s}^{i+1}\right)}(x)\right\}$ is $y_{s^{\prime}-1}$. Claim follows. $\square$ (Claim 17)

By inductively applying above claim we get,

Corollary 18 For all $s$ and for all $i<j \leq n+1, \operatorname{card}\left(\left\{x \mid \varphi_{p\left(q_{s}^{i}\right)}(x) \downarrow \neq\right.\right.$ $\left.\left.\varphi_{p\left(q_{s}^{j}\right)}(x)\right\}\right) \leq j-i$.

Let $C \leq n+1$, be the least value such that $C_{s}=C$ for infinitely many $s$. Thus, for $i \leq C, \lim _{s \rightarrow \infty} q_{s}^{i} \downarrow$, and for $C<i \leq n+1, \lim _{s \rightarrow \infty} q_{s}^{i \uparrow}$. For $i \leq C$, let $q^{i}=\lim _{s \rightarrow \infty} q_{s}^{i}$. Claim 16(c) and invariant (B) thus imply that $\varphi_{p\left(q^{C}\right)}$ is total. Let $f=\varphi_{p\left(q^{C}\right)}$. Note that for all $x \in Z_{f}, f(x)=p(x)$ and $x=q_{s}^{j}$, for some $s \in N$ and $j \leq n+1$ (by construction).

Claim $19 f \in \mathcal{C}$.

Proof. Suppose $q_{s}^{j}>q^{C}$, and $j \leq n+1$. Then, we must have $j>C$, and thus by Claim $16(\mathrm{~d}), q_{s}^{C}=q^{C}$. Thus, by Corollary 18 we have that, for all $q_{s}^{j}>q^{C}, \varphi_{p\left(q^{C}\right)}=^{j-C} \varphi_{p\left(q_{s}^{j}\right)}$. Thus $f \in \mathcal{C} . \square($ Claim 19)

Let $s$ be such that, for all $s^{\prime} \geq s, q_{s}^{C}=q^{C}$. Let $E^{C}=\bigcup_{s^{\prime} \geq s} E_{s}^{C}$. Note that $E^{C}$ is infinite.

Claim 20 For all $k \in E^{C}$, for all $x \in X_{k}, \varphi_{\mathbf{M}(f[(n+2) \cdot k+1])}(x) \neq f(x)$.

ProOF. For all $k \in E^{C}, \varphi_{\mathbf{M}(f[(n+2) \cdot k+1])}$ converges on at most $n+1-C$ elements in $X_{k}$ (otherwise step 3 in the construction would make $C_{s^{\prime}}<C$, for some $\left.s^{\prime}>s\right)$. Moreover, due to invariant (C), for all $k \in E^{C}, \varphi_{\mathbf{M}(f[(n+2) \cdot k+1)]}$ makes at least $n+1-C$ convergent errors in $X_{k}$. It follows that, for $k \in E^{C}$, for each $x \in X_{k}, \varphi_{\mathbf{M}(f[(n+2) \cdot k+1])}(x) \neq f(x) . \square($ Claim 20)

Since, $E^{C}$ is infinite it follows from above claim that $\mathbf{M}$ does not $\mathbf{B c}_{n+1^{-}}^{n}$ identify $f$. Theorem follows. $\square$ (Theorem 15)

As a corollary to the above theorem, if one looks at the errors committed by a general purpose machine on the next $n$ inputs, then for infinitely many 
functions, at infinitely many positions, the machine commits $n$ errors in predicting the next $n$ inputs. Hence, there is an ostensibly unpleasant cost to general purpose learning. However, as we shall see, this can be assuaged at least in some interesting respects (Theorems 28 and 30 in Section 5 below).

Corollary 21 Suppose $m>n$ and $m^{\prime}>n^{\prime}$. If $n>n^{\prime}$, then $\mathbf{B c}_{m}^{n}-\mathbf{B c}_{m^{\prime}}^{n^{\prime}} \neq \emptyset$.

Proof. Theorem 15 shows that $\mathbf{B} \mathbf{c}^{n^{\prime}+1}-\mathbf{B c}_{n^{\prime}+1}^{n^{\prime}} \neq \emptyset$. Now since $\mathbf{B} \mathbf{c}^{n^{\prime}+1} \subseteq$ $\mathbf{B} \mathbf{c}^{n} \subseteq \mathbf{B} \mathbf{c}_{m}^{n}$ (latter inclusion by Proposition 6) and $\mathbf{B} \mathbf{c}_{m^{\prime}}^{n^{\prime}} \subseteq \mathbf{B c}_{n^{\prime}+1}^{n^{\prime}}$ (by Proposition 9), Corollary follows.

Corollary 22 Suppose $m^{\prime}>n^{\prime}$ and $m>n>0$. If $m^{\prime}-n^{\prime}>m-n$, then $\mathbf{B c}_{m}^{n}-\mathbf{B c}_{m^{\prime}}^{n^{\prime}} \neq \emptyset$.

Proof. If $n^{\prime}<n$, then corollary follows from Corollary 21. So suppose $n \leq n^{\prime}$. Theorem 11 shows that $\mathbf{B c}_{m-n+1}^{1}-\mathbf{B c}_{m^{\prime}}^{m^{\prime}-m+n-1} \neq \emptyset$ (note that $\left.m^{\prime}>m-n+1\right)$. Now, $\mathbf{B} \mathbf{c}_{m}^{1+n-1} \supseteq \mathbf{B c}_{m-n+1}^{1}$ (by Proposition 7 ), and $\mathbf{B} \mathbf{c}_{m^{\prime}}^{n^{\prime}} \subseteq$ $\mathbf{B c}_{m^{\prime}}^{m^{\prime}-m+n-1}$ (since $n^{\prime} \leq m^{\prime}-m+n-1$, and by Proposition 8). Corollary follows.

The following corollary resolves all relationships among the $\mathbf{B c}_{m}^{n}$-criteria.

Corollary 23 Suppose $m>n$ and $m^{\prime}>n^{\prime}$. Then: $\mathbf{B c}_{m}^{n} \subseteq \mathbf{B c}_{m^{\prime}}^{n^{\prime}}$ iff $[n=0$ or $\left[n^{\prime} \geq n\right.$ and $\left.\left.m^{\prime}-n^{\prime} \leq m-n\right]\right]$.

PRoOF. If $n>n^{\prime}$ then Corollary 21 shows that $\mathbf{B c}_{m}^{n}-\mathbf{B c}_{m^{\prime}}^{n^{\prime}} \neq \emptyset$. If $m^{\prime}-n^{\prime}>$ $m-n$ and $n>0$, then Corollary 22 shows that $\mathbf{B c}_{m}^{n}-\mathbf{B c}_{m^{\prime}}^{n^{\prime}} \neq \emptyset$.

If $n=0$, then $\mathbf{B c}_{m}^{n}=\mathbf{B c} \subseteq \mathbf{B c}_{m^{\prime}}^{n^{\prime}}$.

If $n^{\prime} \geq n$ and $m^{\prime}-n^{\prime} \leq m-n$, then $\mathbf{B c}_{m}^{n} \subseteq \mathbf{B c}_{m+n^{\prime}-n}^{n^{\prime}}$ (by Proposition 7) and $\mathbf{B c}_{m+n^{\prime}-n}^{n^{\prime}} \subseteq \mathbf{B} \mathbf{c}_{m^{\prime}}^{n^{\prime}}$ (by Proposition 9). Corollary follows.

Corollary 24 For all $m>n, \mathcal{R} \notin \mathbf{B c}_{m}^{n}$.

Thus, no general purpose learning machine can guarantee that anomalies are not concentrated in the near future.

\section{Desirable Properties Achievable By General Purpose Learners}

Since the errors committed by programs output by a general purpose learner can be arbitrarily bad, we look at how this may be assuaged for suitable general purpose learners, and we also determine some additional nice properties a general purpose learner can satisfy. 
On can think of a program for a computable function as a predictive explanation for the function's I/O behavior [BB75,CS83]. Popper's Refutability Principle [Pop68] essentially says that explanations with mistakes should be refutable. As pointed out in [CS83] (see also [CJNM94]), an erroneous predictive explanation (program) for a computable function satisfies Popper's Principle if it computes a total function. ${ }^{2}$ The following theorem says that one can construct a general purpose learner which, on computable function input, almost always outputs programs for total functions; hence, it almost always outputs predictive explanations which satisfy Popper's Principle.

Theorem 25 There exists a machine $\mathbf{M}$, such that, for all $f \in \mathcal{R}$, (i) $\mathbf{M}$ $\mathbf{B c}^{*}$-identifies $f$, and (ii) $\left(\forall^{\infty} n\right)\left[\varphi_{\mathbf{M}(f[n])} \in \mathcal{R}\right]$.

Proof. Define $\mathbf{M}$ as follows. $\mathbf{M}$ on $f[n]$, outputs a program $p_{n}$ such that $\varphi_{p_{n}}$ may be defined as follows.

$\varphi_{p_{n}}(x)$

1. Let $S_{x}=\left\{p \leq n \mid f[n] \subseteq \varphi_{p, x}\right\}$.

2. If $S_{x}=\emptyset$, then let $\varphi_{p_{n}}(x)=0$.

3. Else let $p=\min \left(S_{x}\right)$.

4. Dovetail steps 5 and 6 until one of them succeeds. If step 5 succeeds, before step 6 (if ever) then go to step 7. If step 6 succeeds, before step 5 (if ever) then go to step 8.

5. Search for $s$ such that $\varphi_{p, s}(x) \downarrow$.

6. Search for $p^{\prime}<p$, and $s$ such that, $(\forall y<n)\left[\varphi_{p^{\prime}, s}(y)=f(y)\right]$ and $\varphi_{p^{\prime}, s}(x) \downarrow$.

7. Let $\varphi_{p_{n}}(x)=\varphi_{p}(x)$, and halt.

8. Let $\varphi_{p_{n}}(x)=\varphi_{p^{\prime}}(x)$.

End

We claim that above $\mathbf{M}$ witnesses the theorem. Suppose $f \in \mathcal{R}$. Let $q$ be the least program for $f$. Let $m>q$ be large enough so that, for all $q^{\prime}<q$, there exists a $y<m$ such that $\varphi_{q^{\prime}}(y) \neq f(y)$. Let $p_{n}=\mathbf{M}(f[n])$, and consider the definition of $\varphi_{p_{n}}$ above.

Claim $26(\forall n>m)\left[\varphi_{p_{n}}=^{*} f\right]$. Thus, M Bc* ${ }^{*}$-identifies $f$.

Proof. Note that for large enough $x, \min \left(S_{x}\right)=q$. Also, by definition of $m$, search in step 6 cannot succeed for any $p^{\prime}<p=q$. Also, for $p=q$, there exists an $s$ such that search in step 5 succeeds. Thus, for large enough $x$, $\varphi_{p_{n}}(x)=\varphi_{q}(x)=f(x) . \square($ Claim 26)

Claim $27(\forall n>m)\left[\varphi_{p_{n}} \in \mathcal{R}\right]$.

$\overline{2}$ Then the halting problem [Rog67] does not stand in the way of algorithmically locating the mistakes. 
Proof. Note that, for $n>m, \min \left(S_{x}\right) \geq q$. Thus, for all $x$, step 6 of $\varphi_{p_{n}}(x)$ would eventually succeed since $p^{\prime}=q$ and large enough $s$ satisfy the requirement for success of step 6 . Thus, $\varphi_{p_{n}}(x)$ would be defined at step 8 (if not earlier defined due to step 2 or 7 ). $\square$ (Claim 27)

Theorem follows from above claims. $\square($ Theorem 25)

The following shows that even though a general purpose machine may be locally bad for infinitely many positions, one can ensure that these bad positions have low density.

Theorem 28 For all $n$, there exists a machine $\mathbf{M}$ such that,

(a) $\mathbf{M} \mathbf{B c}^{*}$-identifies $\mathcal{R}$, and

(b) for all $f \in \mathcal{R}, \lim _{x \rightarrow \infty} \frac{\operatorname{card}\left(\left\{k \mid k \leq x \wedge(\forall z \leq n)\left[\varphi_{\mathbf{M}(f[k])}(k+z)=f(k+z)\right]\right\}\right)}{x+1}=1$.

\section{PROOF.}

Suppose $\mathbf{M}^{\prime} \mathbf{B} \mathbf{c}^{*}$-identifies $\mathcal{R}$. Suppose $h$ is a monotonic non-decreasing computable function such that $\lim _{x \rightarrow \infty} \frac{h(x)}{x}=0$. Let $\mathbf{M}$ be defined as follows.

$\mathbf{M}(f[m])$ outputs a program $p_{m}$ defined as follows.

$\varphi_{p_{m}}(y)$

1. If $y<m$, then $\varphi_{p_{m}}(y)=f(y)$.

2. If $y>m+n$, then $\varphi_{p_{m}}(y)=\varphi_{\mathbf{M}^{\prime}(f[m])}(y)$.

3. If $m \leq y \leq m+n$, then search for a $p<h(m)$ such that $f[m] \subseteq \varphi_{p}$, and End $\varphi_{p}(y) \downarrow$. If and when such a $p$ is found, let $\varphi_{p_{m}}(y)=\varphi_{p}(y)$.

Due to step 2, $\varphi_{\mathbf{M}(f[m])}={ }^{*} \varphi_{\mathbf{M}^{\prime}(f[m])}$. Thus, $\mathbf{M} \mathbf{B c}^{*}$-identifies $\mathcal{R}$. Now fix $f \in \mathcal{R}$. Fix $x$. Consider $m \leq x$ such that $\varphi_{\mathbf{M}(f[m])}(m+z) \neq f(m+z)$, for some $z \leq n$. For these $m$ one of the following two conditions must hold.

Case 1: For all $p<h(m),\left[f[m] \nsubseteq \varphi_{p}\right.$ or $\varphi_{p}(m+z) \uparrow$ for some $\left.z \leq n\right]$.

Case 2: There exists a $p<h(m)$ such that $f[m] \subseteq \varphi_{p}$ and $\varphi_{p}(m+z) \downarrow \neq$ $f(m+z)$, for some $z \leq n$.

Case 1 can hold for only for $h(m) \leq \operatorname{MinProg}(f)$. Each $p<h(x)$, can result in Case 2 for at most $n+1$ different $m$ (since if $w$ is the least number such that $\varphi_{p}(w) \neq f(w)$, then for Case 2 to happen, $\left.w-n \leq m \leq w\right)$. Thus, Case 2 can happen for a total of at most $h(x) *(n+1)$ different $m \leq x$. Let $c$ be such that $h(c)>\operatorname{MinProg}(f)$. Thus, Case 1 or Case 2 can happen for at 
most $c+h(x) *(n+1)$ different $m$. Now, $\lim _{x \rightarrow \infty} \frac{c+h(x) *(n+1)}{x+1}=0$ (since $c$ and $n$ are constants and $\left.\lim _{x \rightarrow \infty} h(x) / x=0\right)$. Since $f$ was arbitrary computable function, theorem follows.

Since general purpose learners are always quite erroneous (of course the density of erroneous, near future intervals can be made small), it is interesting to consider which classes a general purpose learner may additionally identify in a better or stricter sense.

\section{Definition 29 [Min76,BB75,CJNM94]}

(a) $\mathbf{M}$ is said to be reliable iff, for all $f$ such that $\mathbf{M}(f) \downarrow, \mathbf{M} \mathbf{E x}$-identifies $f$.

(b) $\mathbf{M}$ is said to reliably $\mathbf{E x}$-identify $\mathcal{C}$, iff $\mathbf{M}$ is reliable and $\mathbf{M} \mathbf{E x}$-identifies $\mathcal{C}$.

(c) $\operatorname{RelEx}=\{\mathcal{C} \mid$ some machine reliably Ex-identifies $\mathcal{C}\}$.

Intuitively, reliable machines do not deceive us by converging falsely. As noted above, r.e. classes of computable functions (such as the class of polynomial functions and the class of primitive recursive functions) as well as the class of total run time functions can be reliably Ex-identified.

The following theorem shows that for any class $\mathcal{S}$ in RelEx, one can create a general purpose learning machine which Ex-identifies $\mathcal{S}$ !

Theorem 30 Suppose $\mathcal{S} \in \mathbf{R e l E x}$. Then there exists an $\mathbf{M}$ such that $\mathbf{M}$ $\mathbf{B c}^{*}$-identifies $\mathcal{R}$ and $\mathbf{E x}$-identifies $\mathcal{S}$.

Proof.

Suppose $\mathbf{M}_{H}$ is a machine which $\mathbf{B} \mathbf{c}^{*}$-identifies $\mathcal{R}$. Below $P$ ranges over finite sets of programs. Let Prog be a recursive function such that $\varphi_{\operatorname{Prog}(P, f[n])}$ may be defined as follows.

$\varphi_{\operatorname{Prog}(P, f[n])}(x)$

1. If there exists a $y \leq x$ and $i, j \in P$ such that $\Phi_{i}(y) \leq x, \Phi_{j}(y) \leq x$ and $\varphi_{i}(y) \neq \varphi_{j}(y)$.

Then let $\varphi_{\operatorname{Prog}(P, f[n])}(x)=\varphi_{\mathbf{M}_{H}(f[n])}(x)$.

2. Else, search for an $i \in P$ such that $\varphi_{i}(x) \downarrow$. If and when such an $i$ is found, End let $\varphi_{\operatorname{Prog}(P, f[n])}(x)=\varphi_{i}(x)$.

Claim 31 For all $f \in \mathcal{R}$, for all $n$, for all $P$, 
$\left[(\exists p \in P)\left[\varphi_{p}=f\right] \wedge(\forall p \in P)\left[\varphi_{p} \subseteq f\right]\right] \Rightarrow\left[\varphi_{\operatorname{Prog}(P, f[n])}=f\right]$.

Proof. Fix $f \in \mathcal{R}$, and $n \in N$. Suppose, $(\exists p \in P)\left[\varphi_{p}=f\right] \wedge(\forall p \in$ $P)\left[\varphi_{p} \subseteq f\right]$. Fix any input $x$. Since $(\forall p \in P)\left[\varphi_{p} \subseteq f\right]$, 'If' clause in step 1 of $\varphi_{\operatorname{Prog}(P, f[n])}(x)$ fails. Also, there exists a $p^{\prime} \in P$, such that $\varphi_{p^{\prime}}(x) \downarrow$. It follows that search in step 2 of $\varphi_{\operatorname{Prog}(P, f[n])}(x)$, succeeds. Thus, $\varphi_{\operatorname{Prog}(P, f[n])}(x) \downarrow$. $\varphi_{\operatorname{Prog}(P, f[n])}(x)=f(x)$ now follows from the hypothesis that $(\forall p \in P)\left[\varphi_{p} \subseteq f\right]$. $\square$ (Claim 31)

Claim 32 For all $f \in \mathcal{R}$, for all but finitely many $n$, for all $P$,

$(\exists p \in P)\left[\varphi_{p}=f\right] \Rightarrow\left[\varphi_{\operatorname{Prog}(P, f[n])}=^{*} f\right]$.

Proof.

Fix $f \in \mathcal{R}$. Let $n_{0}$ be such that, for all $n \geq n_{0}, \varphi_{\mathbf{M}_{H}(f[n])}={ }^{*} f$.

Suppose there exists a $p \in P$ such that $\varphi_{p}=f$. If, $(\forall p \in P)\left[\varphi_{p} \subseteq f\right]$, then by Claim $31 \varphi_{\operatorname{Prog}(P, f[n])}=f$.

If, $(\exists p \in P)\left[\varphi_{p} \not \subset f\right]$, then using $(\exists p \in P)\left[\varphi_{p}=f\right]$, it follows that, there exist $i, j \in P, x_{0}, y_{0} \in N$, such that $y_{0} \leq x_{0}$, and $\Phi_{i}\left(y_{0}\right) \leq x_{0}, \Phi_{j}\left(y_{0}\right) \leq x_{0}$ and $\varphi_{i}\left(y_{0}\right) \neq \varphi_{j}\left(y_{0}\right)$. Thus, for all $x \geq x_{0}, \varphi_{\operatorname{Prog}(P, f[n])}(x)=\varphi_{\mathbf{M}_{H}(f[n])}(x)$. It follows that, for $n \geq n_{0}, \varphi_{\operatorname{Prog}(P, f[n])}={ }^{*} \varphi_{\mathbf{M}_{H}(f[n])}={ }^{*} f$. Claim follows. $\square$ (Claim 32)

We now show the theorem using above claims. Suppose $\mathbf{M}^{\prime}$ RelEx-identifies $\mathcal{S}$. Without loss of generality assume $\mathcal{S}=\mathbf{E x}\left(\mathbf{M}^{\prime}\right)$, and for all $n, \mathbf{M}^{\prime}(f[n]) \leq n$. Let $\mathrm{SB}(f[n])=\max \left(\left\{\mathbf{M}^{\prime}(f[x]), \operatorname{card}\left(\left\{m<n-1 \mid \mathbf{M}^{\prime}(f[m]) \neq \mathbf{M}^{\prime}(f[m+\right.\right.\right.\right.$ $1])\})\}$ ). Note that if $\mathbf{M}^{\prime}(f) \downarrow$, then $\lim _{n \rightarrow \infty} \mathrm{SB}(f[n]) \downarrow \geq \mathbf{M}^{\prime}(f)$; if $\mathbf{M}^{\prime}(f) \uparrow$, then $\liminf _{n \rightarrow \infty} \mathrm{SB}(f[n])=\infty$. Also $\mathrm{SB}(f[n]) \leq n$.

Now $\mathbf{M}(f[n])$ outputs $\operatorname{Prog}\left(P_{n}^{f}, f[\mathrm{SB}(f[n])]\right)$, where $P_{n}^{f}=\{i \leq \mathrm{SB}(f[n]) \mid$ $\left.\varphi_{i, n} \subseteq f\right\}$.

Claim 33 If $f \in \mathcal{S}$ then $\mathbf{M}$ Ex-identifies $f$.

Proof.

Suppose $f \in \mathcal{S}$. Thus, $\lim _{n \rightarrow \infty} \mathrm{SB}(f[n]) \downarrow$. Let $\mathrm{SB}(f)$ denote $\lim _{n \rightarrow \infty} \mathrm{SB}(f[n])$. Note that $\operatorname{SB}(f) \geq \operatorname{MinProg}(f)$. Let $n_{0}$ be such that, for all $n \geq n_{0}$, $\mathrm{SB}(f[n])=\mathrm{SB}(f)$, and $\left(\forall p \leq \mathrm{SB}(f) \mid \varphi_{p} \not \subset f\right)\left[\varphi_{p, n_{0}} \not f f\right]$. It follows that for all $n \geq n_{0}, P_{n}^{f}=\left\{p \leq \mathrm{SB}(f) \mid \varphi_{p} \subseteq f\right\}$. Since $\operatorname{MinProg}(f) \leq \mathrm{SB}(f)$, it follows that $\operatorname{MinProg}(f) \in P_{n}^{f}$. Thus, $\mathbf{M}(f) \downarrow=\operatorname{Prog}\left(P_{n_{0}}^{f}, f[\mathrm{SB}(f)]\right)$. Now, using Claim 31 we have that $\varphi_{\operatorname{Prog}\left(P_{n}^{f}, f[\mathrm{SB}(f)]\right)}=f$. Claim follows. $\square$ (Claim 33)

Claim 34 If $f \in \mathcal{R}-\mathcal{S}$, then $\mathbf{M} \mathbf{B c}^{*}$-identifies $f$. 
Proof. Suppose $f \in \mathcal{R}-\mathcal{S}$. Then, $\liminf _{n \rightarrow \infty} \operatorname{SB}(f[n])=\infty$. Now fix $n_{0}$ such that

(i) for all $n \geq n_{0}, \operatorname{SB}(f[n]) \geq \operatorname{MinProg}(f)$, and

(ii) for $n \geq n_{0}$, for all $P,(\exists p \in P)\left[\varphi_{p}=f\right] \Rightarrow\left[\varphi_{\operatorname{Prog}(P, f[n])}=^{*} f\right]$.

Note that by Claim $32, n_{0}$ satisfying (ii) exists. Note that by (i), and definition of $P_{n}^{f}$, for all $n \geq n_{0}, P_{n}^{f}$ contains $\operatorname{MinProg}(f)$.

It follows from (ii) that, for all $n \geq n_{0},\left[\varphi_{\operatorname{Prog}(P, f[n])}=^{*} f\right]$. This proves the claim. $\square$ (Claim 34)

Theorem follows from Claim 33 and 34. $\square($ Theorem 30)

On the other hand, Corollary 36 below shows that RelEx cannot be replaced by finite identification.

Definition 35 [Gol67]

(a) M finitely-identifies $f$, iff there exists an $n$ and a $p$ such that, $\varphi_{p}=f$, $\mathbf{M}(f[m])=$ ?, for $m<n$, and $\mathbf{M}(f[m])=p$, for $m \geq n$.

(b) $\mathbf{M}$ finitely-identifies $\mathcal{C}$, iff $\mathbf{M}$ finitely-identifies each $f \in \mathcal{C}$.

(c) $\mathcal{C}$ is finitely-identifiable iff some $\mathbf{M}$ finitely-identifies $\mathcal{C}$.

Let $\mathcal{S}_{0}=\left\{f \mid \varphi_{f(0)}=f\right\}$. Note that $\mathcal{S}_{0}$ is finitely-identifiable.

Corollary 36 Suppose $n \in N$ and $\mathbf{M} \mathbf{B c}^{*}$-identifies $\mathcal{R}$. Then there exists an $f \in \mathcal{S}_{0}$ such that $\mathbf{M}$ does not $\mathbf{B c}^{n}$-identify $f$.

Proof. Suppose $\mathbf{M}$ is as given in the hypothesis. Let $g(x)=n$. Then, proof of Theorem 4 gives an $f \in \mathcal{S}_{0}$, such that for infinitely many $x, \varphi_{\mathbf{M}(f[x])} \neq^{n}$ $f$.

It would be interesting to study what other useful properties a suitable general purpose learner can be made to satisfy.

\section{Conclusions}

Harrington [CS83] surprisingly constructed a general purpose learner, i.e., a machine which $\mathbf{B c}^{*}$-identifies all the computable functions. However, the programs output by Harrington's machine become more and more degenerate, 
i.e., in general, the finite set of anomalies in each final program grows without bound. In this paper we showed that this is unavoidable (Theorem 4 above).

Since the programs output by any general purpose learning machine make large number of errors on infinitely many functions, it is interesting to study how these errors are or can be distributed. Based on this motivation we defined new criteria of inference called $\mathbf{B c}_{m}^{n}$, and completely resolved the relationship between different $\mathbf{B} \mathbf{c}_{m}^{n}$ criteria of inference. Among other results, we showed that any general purpose learning machine is poor in predicting near future values. In particular any general purpose learning machine $\mathbf{M}$ predicts the next $n$ values wrongly infinitely often. In contrast, though, we show that the density of such bad prediction points can be made vanishingly small (Theorem 28 above).

We constructed a general purpose learning machine $\mathbf{M}$ such that, on any computable function input, all but finitely many of the programs output by $\mathbf{M}$ are for total functions. Hence, almost all of its conjectures satisfy Popper's Refutability Principle.

We also showed that for every class of computable functions, $\mathcal{S}$, which can be Ex-identified by a reliable machine [Min76,BB75,CJNM94] (see definition in Section 5 above), some general purpose learning machine additionally Exidentifies $\mathcal{S}$. We further show, though, that reliable identification in the just above statement cannot be replaced by finite identification.

It would be interesting to study which other useful properties a general purpose learner can or cannot have.

\section{References}

[Bār71] J. Bārzdinsš. Complexity and Frequency Solution of Some Algorithmically Unsolvable Problems. PhD thesis, Novosibirsk State University, 1971. In Russian.

[Bār74] J. Bārzdiņš. Two theorems on the limiting synthesis of functions. In Theory of Algorithms and Programs, vol. 1, pages 82-88. Latvian State University, 1974. In Russian.

[BB75] L. Blum and M. Blum. Toward a mathematical theory of inductive inference. Information and Control, 28:125-155, 1975.

[Blu67] M. Blum. A machine-independent theory of the complexity of recursive functions. Journal of the ACM, 14:322-336, 1967.

[Cas74] J. Case. Periodicity in generations of automata. Mathematical Systems Theory, 8:15-32, 1974. 
[CJNM94] J. Case, S. Jain, and S. Ngo Manguelle. Refinements of inductive inference by Popperian and reliable machines. Kybernetika, 30:23-52, 1994.

[CS83] J. Case and C. Smith. Comparison of identification criteria for machine inductive inference. Theoretical Computer Science, 25:193-220, 1983.

[FBW98] R. Freivalds, O. Botuscharov, and R. Wiehagen. Identifying nearly minimal Gödel numbers from additional information. Annals of Mathematics and Artificial Intelligence, 23:199-209, 1998.

[FW79] R. Freivalds and R. Wiehagen. Inductive inference with additional information. Journal of Information Processing and Cybernetics (EIK), 15:179-195, 1979.

[Gol67] E. M. Gold. Language identification in the limit. Information and Control, 10:447-474, 1967.

[JORS99] S. Jain, D. Osherson, J. Royer, and A. Sharma. Systems that Learn: An Introduction to Learning Theory. MIT Press, Cambridge, Mass., second edition, 1999.

[Min76] E. Minicozzi. Some natural properties of strong identification in inductive inference. Theoretical Computer Science, pages 345-360, 1976.

[Pod74] K. Podnieks. Comparing various concepts of function prediction, Part I. In Theory of Algorithms and Programs, vol. 1, pages 68-81. Latvian State University, Riga, Latvia, 1974.

[Pop68] K. Popper. The Logic of Scientific Discovery. Harper Torch Books, New York, second edition, 1968.

[Ric80] G. Riccardi. The Independence of Control Structures in Abstract Programming Systems. PhD thesis, SUNY/Buffalo, 1980.

[Ric81] G. Riccardi. The independence of control structures in abstract programming systems. Journal of Computer and System Sciences, 22:107-143, 1981.

[Rog58] H. Rogers. Gödel numberings of partial recursive functions. Journal of Symbolic Logic, 23:331-341, 1958.

[Rog67] H. Rogers. Theory of Recursive Functions and Effective Computability. McGraw-Hill, 1967. Reprinted by MIT Press in 1987.

[Roy87] J. Royer. A Connotational Theory of Program Structure, volume 273 of Lecture Notes in Computer Science. Springer-Verlag, 1987. 